\title{
Medical Image Fusion based on Shearlets and Human Feature Visibility
}

\author{
Nemir Ahmed Al-Azzawi \\ Baghdad University \\ Biomedical Engineering Department
}

\begin{abstract}
Medical image fusion is a technique that integrates complementary information from multimodality images. The fused image is more suitable for treatment plan strategies. In this paper, an efficient medical image fusion method has been proposed based on shearlet transform and human visibility feature as fusion rule. Image fusion rule is the solution that influences the quality of image fusion. The multimodal medical images were first decomposed using the shearlet transform then fusion rules were applied to shearlet coefficients. The low-frequency coefficients are fused by human visibility feature method. While, the high frequency coefficients are fused by the maximum selection fusion rule. The final fusion image is obtained by directly applying inverse shearlet transform to the fused coefficients. The technique proposed has successfully been used in CT/MRI image fusion for tumor diagnosis. The visual experiments and quantitative assessments demonstrate the effectiveness of this method compared to present image fusion.
\end{abstract}

\section{Keywords}

Shearlet transform; medical image fusion; human visibility feature; multimodality; CT/MRI image.

\section{INTRODUCTION}

In recent years, the improvement of medical treatment procedure, medical images fusion being used further in the diagnosing diseases, tumor tissues analysis and treatment plain strategies etc. Medical image fusion is the procedure of combining complementary information of a particular organ focused by the different types of modalities [1]. The medical images can include X-ray, computed tomography (CT), magnetic resonance imaging (MRI), magnetic resonance angiography (MRA), and positron emission tomography (PET) images. For example, fusing MR and CT images is a benefit for the operational results in computer assisted navigated neurosurgery of temporal bone tumors[2]. PET/CT in lung cancer, MRI/PET in brain tumors, SPECT/CT in abdominal studies and ultrasound images/MRI for vascular blood flow [3]. The popular fusion methods are based on multiresolution analysis, such as the discrete wavelet transform [4], stationary wavelet transform [5] and contourlet transform [6]

Conversely, due to the technique limitation, the quality of fused medical images is sometimes unsatisfactory, which degrade the accuracy of human interpretation and further medical image analysis needs to be improved. Recently, a theory for multidimensional data has been developed to provide higher directional sensitivity than wavelets. Extensive researches have been conducted on image fusion techniques, and various fusion algorithms for medical image have been developed depending on the merging stage [7-11].
Medical image fusion usually employs the pixel level fusion techniques. The advantage of pixel fusion is that the images use to contain the original information. Multiscale geometric analysis was introduced by [12] as contrast decomposition scheme that used to relate the luminance processing in the early stages of the human visual system. Shearlets are a multiscale framework which allows to efficiently encoding anisotropic features in multivariate problem classes. Originally, shearlets were introduced in 2006 [13]. The shearlet transform is unlike the traditional wavelet transform which does not possess the ability to detect directionality. One of the most significant properties of shearlets is the information that they provide optimally sparse approximations (in the sense of optimality [14]). An important advantage of the shearlet transform over the contourlet transform is that there are no restrictions on the direction numbers [13, 15-16]. In addition the various methods mentioned, image fusion method based on shearlet transform was recently presented [17]. F. Sillion and G. Drettakis [18], introduced a new approach to controlling error in hierarchical clustering algorithm based on feature visibility. This feature is motivated from the human visual system, and is defined as [19]. Image fusion rule is the key that influences the quality of image fusion. To improve the fused image quality, new methods for fusion rules which depend on human visibility feature of shearlet coefficients have been presented in this paper. The registration technique employed is as described by Al-Azzawi [20].

In this paper, pixel-level fusion algorithm for multimodality medical image based on shearlet transform and human feature visibility system is developed. Feature visibility is included as the fusion rules. Experimental results show that the proposed fusion algorithm provides a successful way to enable more accurate analysis of multimodal images.

\section{SHEARLET TRANSFORM}

The shearlets are a multiscale framework which allows to efficiently encoding anisotropic features in multivariate problem classes. Shearlets are constructed by parabolic scaling, shearing and translation applied to few generating functions [13].

The construction of continuous shearlet systems is based on parabolic scaling matrices $A_{a}=\left(\begin{array}{cc}a & 0 \\ 0 & \sqrt{a}\end{array}\right)$ for $a>0$. The mean is change the resolution, on shear matrices $S_{s}=\left(\begin{array}{ll}1 & 0 \\ 0 & 1\end{array}\right)$ for $s \in \mathbb{R}$. Let $\psi_{1} \in L^{2}(\mathbb{R})$ be a function satisfying the discrete Calderón condition, i.e. :

$$
\sum_{j \in \mathbb{Z}}\left|\hat{\psi}_{1}\left(2^{-j} \xi\right)\right|^{2}=1, \quad \xi \in \mathbb{R}
$$


Where $\hat{\psi}_{1}$ denoted the Fourier transform of $\psi_{1}$. With $\hat{\psi}_{1} \in$ $\mathrm{C}^{\infty}(\mathbb{R})$, supp $\hat{\psi}_{1} \subseteq[-1 / 2,-1 / 16] \cup[1 / 16,1 / 2]$. Furthermore, let $\psi_{2} \in L^{2}(\mathbb{R})$ be such that $\hat{\psi}_{2} \in \mathrm{C}^{\infty}(\mathbb{R})$, supp $\hat{\psi}_{2} \subseteq[-1,1]$ and :

$$
\sum_{k \in \mathbb{Z}}\left|\hat{\psi}_{2}(\xi+k)\right|^{2}=1, \quad \xi \in \mathbb{R}
$$

One typically choose $\hat{\psi}_{2}$ to be smooth function then :

$$
\hat{\psi}(\xi)=\widehat{\psi}_{1}\left(\xi_{1}\right) \widehat{\psi}_{2}\left(\frac{\xi_{2}}{\xi_{1}}\right), \quad \xi=(\xi 1, \xi 2) \in \mathbb{R}^{2}
$$

The $\mathrm{SH}(\psi)$ is called a classical shearlet constitutes a Parseval frame for $L^{2}(\mathbb{R})$ consisting of band-limited functions. It can be shown that the corresponding discrete shearlet system $S H(\phi, \psi, \tilde{\psi} ; c)$, the frequency domain is divided into a lowfrequency part and two conic regions (see Figure 1):

$$
\begin{aligned}
& \mathrm{F}^{\mathrm{L}}=\left\{\left(\xi_{1}, \xi_{2}\right) \in \mathbb{R}^{2}|| \xi_{1}|,| \xi_{2} \mid \leq 1\right\}, \\
& \mathrm{C}_{\mathrm{H}}=\left\{\left(\xi_{1}, \xi_{2}\right) \in \mathbb{R}^{2}|| \xi_{2} / \xi_{1} \mid>1\right\}, \\
& \mathrm{C}_{\mathrm{V}}=\left\{\left(\xi_{1}, \xi_{2}\right) \in \mathbb{R}^{2}|| \xi_{1} / \xi_{2} \mid>1\right\},
\end{aligned}
$$

The associated cone-adapted discrete shearlet system consists of three parts, each one corresponding to one of these frequency domains. It is generated by three functions $\phi, \psi, \tilde{\psi} \in L^{2}(\mathbb{R})$ and a lattice sampling factor $c=\left(c_{1}, c_{2}\right)$ for $\mathbb{R}^{2}>0$. Finally, the scaling function $\phi$ is associated with the low-frequency part $\mathrm{F}^{\mathrm{L}}$.

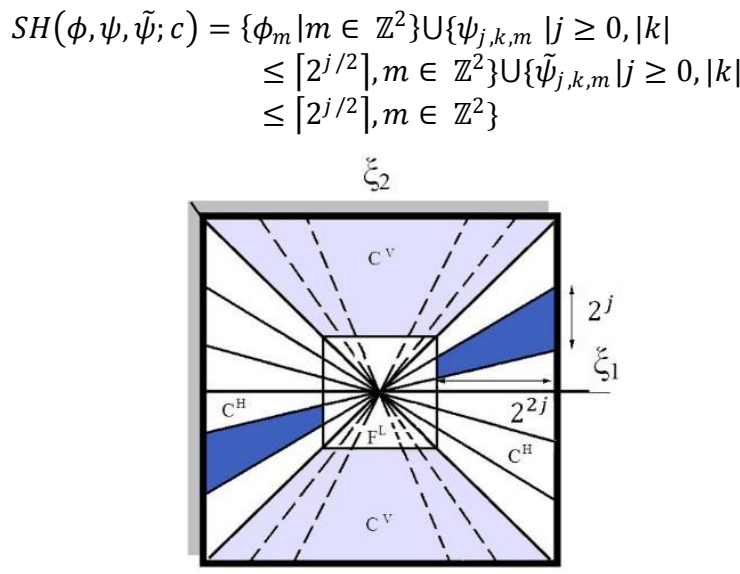

Fig 1: The decomposition of the frequency domain into cones, low-frequency and the parabola scale of the shearlets.

\section{HUMAN FEATURE VISIBILITY}

The concept of human feature visibility is introduced as a method to evaluate the quality of an image. This method is restricted by the fact that the human visual system is influenced by the contrast. Therefore, the visibility of image can be more closely approach to general much better visibility of direct scene perception by the human observer. Local energy has been proposed as a generalized model for feature detection in pre-attentive human vision [21]. Feature visibility method has been employed in previous researches [22-23] as fusion rules. Morrone and Burr [24] noted and exploited this key point in developing a model for feature detection in human vision. In this model, a given image is filtered with a set of orthogonal filter pairs exhibiting even and odd symmetries. The Michelson visibility operator [25] has been used to measure the strength of interference fringes. The visibility feature is formed from an image by extending the Michelson visibility feature operator to two dimensions. Let
$I_{m, n}$ denote an image containing $M$ rows and $N$ columns, for 0 $\leq m \leq M, 0 \leq n \leq N$. To create the local visibility feature. The maximum pixel value $I^{\max }$ and the minimum pixel value $I^{\mathrm{min}}$ are calculated. There are a number of extensions that can be made to the visibility feature operator. These include a second derivative-like visibility feature operator, and various forms of local visibility feature operators:

$V_{m, n}^{I}=\frac{I^{\max }-2 I_{m, n}+I^{\min }}{I^{\max }+2 I_{m, n}+I^{\min }}$

$V_{m, n}^{I}=\sqrt{\left(\frac{I_{m, n}-I^{\min }}{I^{\max }+I^{\min }}\right)\left(\frac{I^{\max }-I_{m, n}}{I^{\max }+I^{\min }}\right)}$

Or as define in [19], this feature is motivated from the human visual system:

$V_{m, n}^{I}=\frac{\left|I_{m, n}-\mu\right|}{\mu^{\alpha}}$

where $\mu$ is the mean intensity value of the image, and $\alpha$ is a visual constant ranging from 0.6 to 0.7 .

\section{PROPOSED ALGORITHM}

The fusion algorithm based on shearlet transform can be divided into three steps. They are decomposition, fusion rules and reconstruction. The fusion framework using shearlet transform is shown in Figure 2.

\subsection{Decomposition}

The source images $\boldsymbol{A}(\mathrm{CT})$ and $\boldsymbol{B}$ (MRI), respectively are decomposed with shearlet transform, and obtain the corresponding coefficients. Both horizontal and vertical cones are adopted in this method. Image decomposition is composed by two parts, decomposition of multi-direction ( $K^{\text {th }}$ directions) and $J$-level multi-scale wavelet packets.

\subsection{Fusion Rules}

The paper adopts the human feature visibility fusion scheme to perform the selection of shearlet low frequency coefficients. The human visual feature is used to provide better details and conform to the human observer. Local mean intensity value of the image can be expressed as:

$\mu^{(A \text { or } B)}(m, n)=\sum_{k, l \in W} W . \operatorname{Coff}_{s h}^{L,(A \text { or } B)}(m, n)$

Where, $W$ is a template of size $k \times l$ and satisfies the normalization rule. Therefore, the human feature visibility of shearlet coefficients is calculated as:

$V^{L,(A \text { or } B)}{ }_{s h}(m, n)=\frac{\left|\operatorname{Coff}_{s h}^{L,(A \text { or } B)}(m, n)-\mu_{m, n}^{(A \text { or } B)}\right|}{\left(\mu_{m, n}^{(A \text { or } B)}\right)^{\alpha}}$

The normalized weight $D^{L,(A \text { or } B)}$ is defined as:

$\begin{aligned} D^{L, A} & =\frac{V^{L, A}{ }_{s h}(m, n)}{V^{L, A}{ }_{s h}(m, n)+V^{L, B}{ }_{s h}(m, n)} \\ D^{L, B} & =\frac{V^{L, B}{ }_{s h}(m, n)}{V^{L, A}{ }_{s h}(m, n)+V^{L, B}{ }_{s h}(m, n)}\end{aligned}$

Thus, the fused image has the same energy distribution as the original input images. The coefficients of low frequency components for fused image $F$ is shown below:

$\operatorname{Coff}_{s h}^{L, F}=\operatorname{Coff}_{s h}^{L, A} \cdot D^{(L, A)}+\operatorname{Coff}_{s h}^{L, B} \cdot D^{(L, B)}$

Where, $\operatorname{Coff}_{s h}^{L, A}$ and $\operatorname{Coff}_{s h}^{L, B}$ represent low frequency coefficients of image $A$ and $B$ respectively. For the coefficients of the high-frequency, we calculate maximum selection scheme [26]. Larger value of coefficients in shearlet domain $\operatorname{Coff}_{s h}^{H,(A \text { or B })}$ means there is more high frequency 
information. Weights $D^{(H, A)}$ and $D^{(H, B)}$ needs to be calculated as:

$$
\begin{aligned}
& D^{(H, A)}=\left\{\begin{array}{ll}
1 & \left|\operatorname{Coff}_{\text {sh }}^{H, A}\right| \geq\left|\operatorname{Coff}_{s h}^{H, B}\right| \\
0 & \text { otherwise }
\end{array}\right\} \\
& D^{(H, B)}=1-D^{(H, A)}
\end{aligned}
$$

The coefficients of high frequency components in shearlet domain for fused image $F$ is,

$$
\operatorname{Coff}_{s h}^{H, F}=\operatorname{Coff}_{s h}^{H, A} \cdot D^{(H, A)}+\operatorname{Coff}_{s h}^{H, B} \cdot D^{(H, B)}
$$

\subsection{Reconstruction}

The modified fused coefficients are reconstructed by inverse shearlet transform to obtain fused image. The corresponding fused image results are given in Figure 4. Four data sets images are selected to prove the validity proposed.

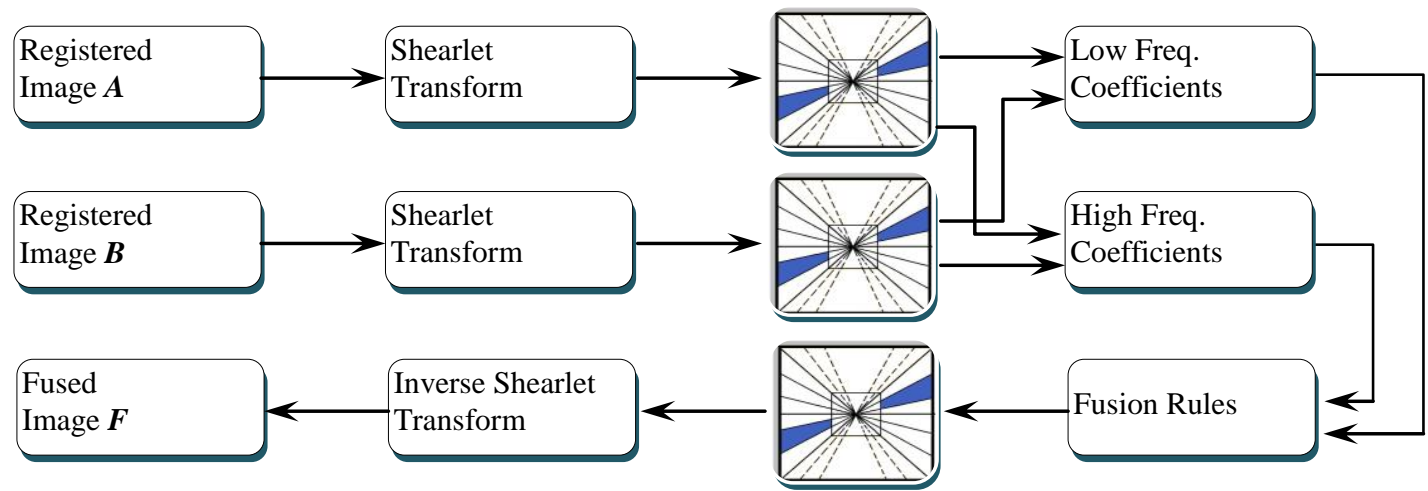

Fig. 2: Block diagram of the proposed image fusion algorithm based on Shearlet.
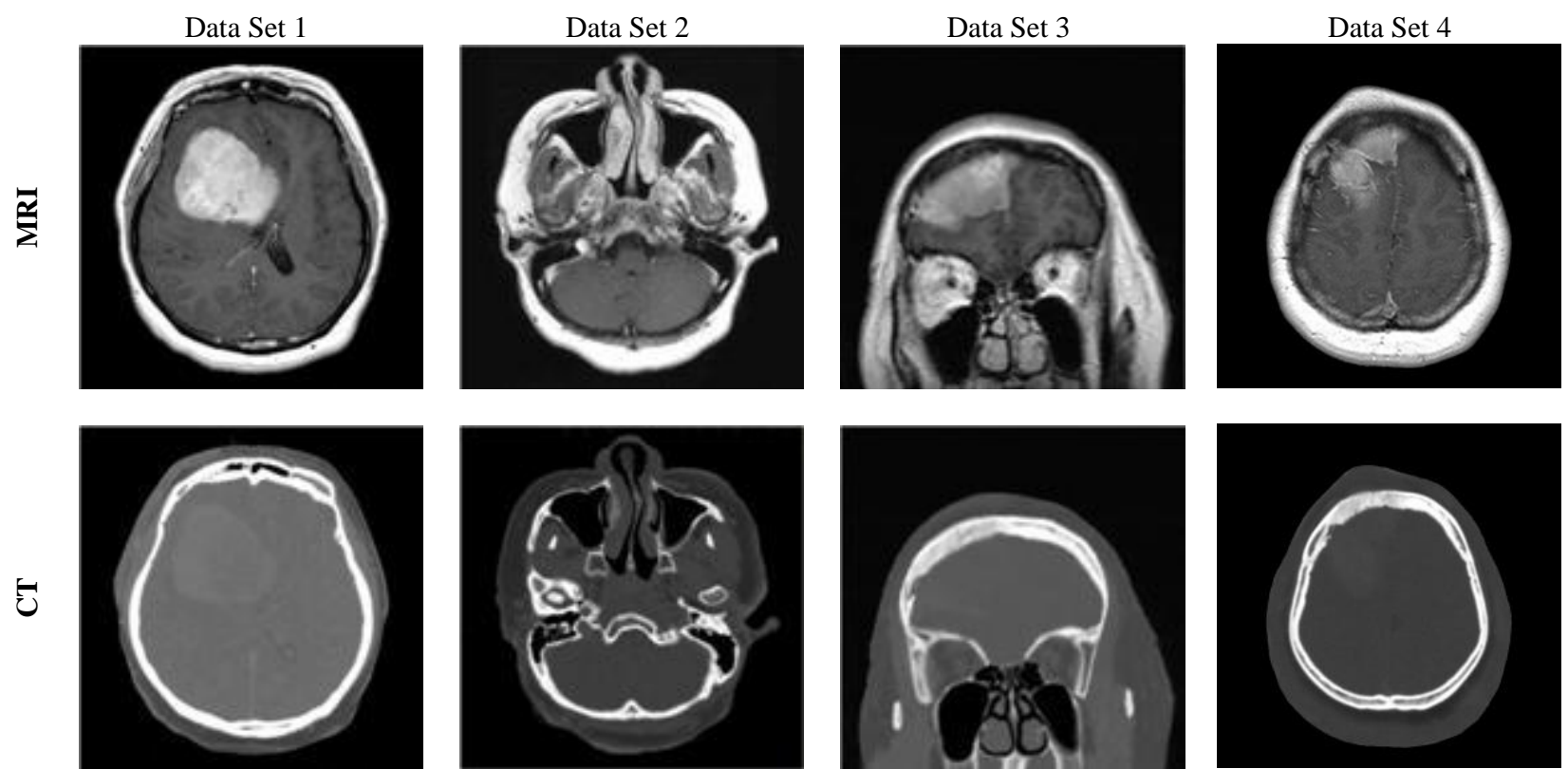

Fig 3 : Original multimodality image dataset 1, 2, 3 and 4. 


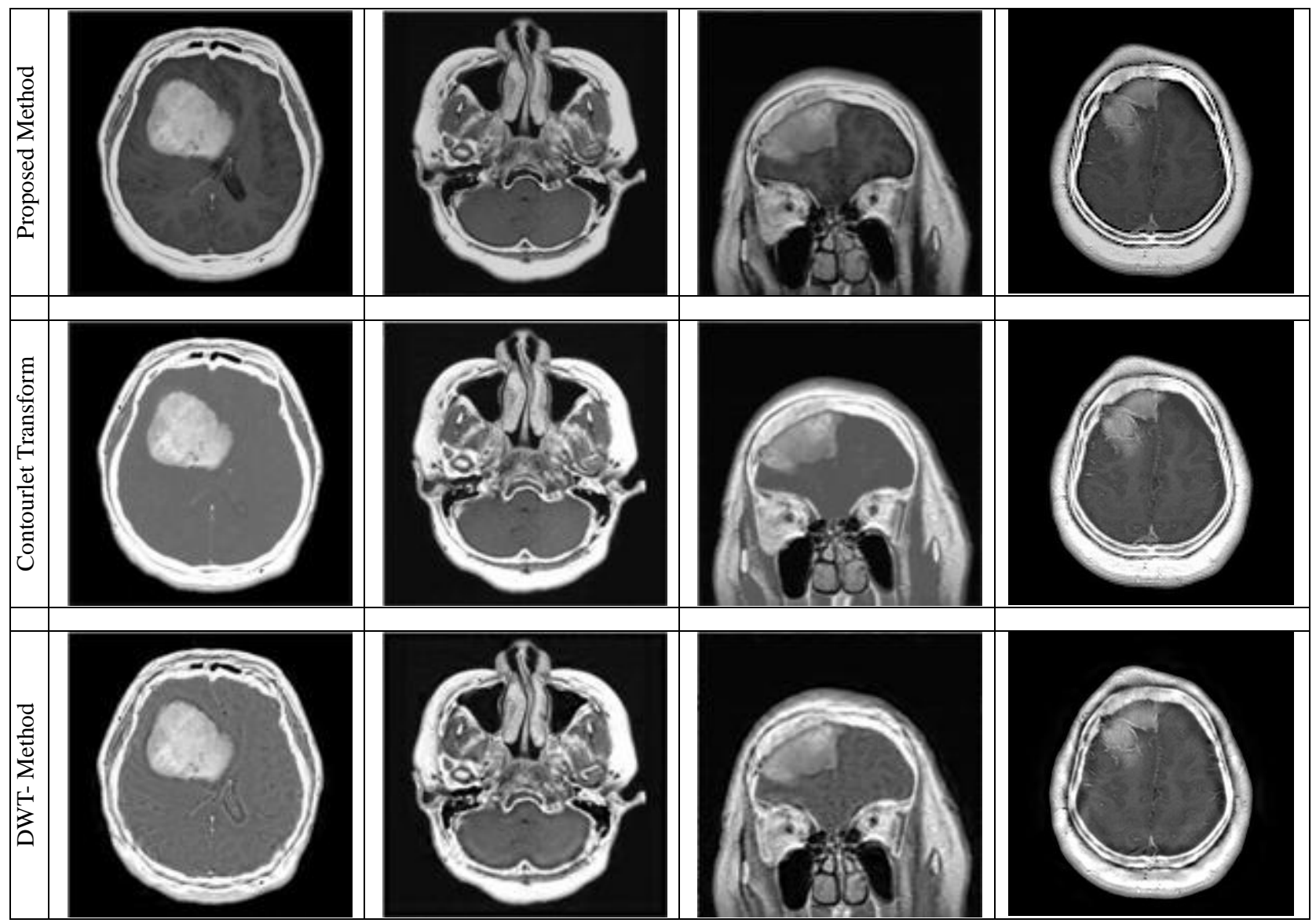

Fig 4: Fusion results on test original multimodality image dataset 1, 2, 3 and 4 using proposed method, contourlet transform and DWT method.

Table 1. Comparison of image quality metrics for fusion algorithms.

\begin{tabular}{|c|c|c|c|c|c|c|c|}
\hline Data Set & Algorithm & EN & STD & OCE & AVG & IQI & RMSE \\
\hline \multirow{3}{*}{ Image set 1 } & Proposed & 7.1961 & 34.8192 & 0.6214 & 0.0795 & 0.3013 & 0.1506 \\
& CT [8] & 6.6424 & 31.1693 & 0.9041 & 0.0765 & 0.2629 & 0.2218 \\
& DWT [4] & 6.5142 & 24.3864 & 0.7274 & 0.0662 & 0.2605 & 0.2336 \\
\hline \multirow{3}{*}{ Image set 2 } & Proposed & 7.6572 & 56.7993 & 0.6394 & 0.0391 & 0.2092 & 0.1164 \\
& CT [8] & 7.1332 & 54.1504 & 0.9748 & 0.0380 & 0.1918 & 0.1662 \\
& DWT [4] & 6.9543 & 47.2304 & 1.0506 & 0.0346 & 0.1603 & 0.2703 \\
\hline \multirow{3}{*}{ Image set 3 } & CT [8] & 6.9351 & 46.6294 & 1.0782 & 0.0262 & 0.1305 & 0.2538 \\
& DWT [4] & 6.6997 & 41.4623 & 1.1278 & 0.0231 & 0.1307 & 0.2889 \\
\hline & Proposed & 6.9467 & 44.2937 & 0.7654 & 0.0521 & 0.3455 & 0.2133 \\
& CT [8] & 6.8824 & 43.1963 & 0.8843 & 0.0433 & 0.2387 & 0.2422 \\
& DWT [4] & 6.5198 & 42.0087 & 1.1076 & 0.0419 & 0.2176 & 0.3142 \\
\hline
\end{tabular}




\section{EXPERIMENTAL RESULTS}

In this section, by fusing CT/MRI images for comparing the performances of proposed medical fusion scheme. The multimodal medical images has been provided by Hospital Universiti Sains Malaysia (HUSM) [1]. Figure 3, shows the original multimodality image dataset $1,2,3$ and 4 . To validate and test the fusion systems, numerous experiments were conducted with images of different dimensions and pixel resolutions. Thirty groups with different subjects of human brain images were selected. An experimental result was compared with method contourlet transform (CT) [8] and discrete wavelet method (DWT) [4]

The subjective visual perception gives us straight comparisons, and various objectives. Quality assessments are also used to evaluate the performance of the proposed algorithm. The following image quality metrics are used in this paper: entropy (EN), standard deviation (STD), overall cross entropy (OCE) [8], average gradient (Avg) [27], image quality index (IQI) [28-29], and root mean squared error (RMSE)

Table 1, shows the experimental results. From indicators the EN and STD are the greatest with the proposed method, higher value of entropy means that more information in certain image. Average gradient (Avg) is used to calculate the performance of the fused image and reflects the clarity of the fused image. The dynamic range of IQI is $[-1,1]$. The best value 1 is achieved if and only if fused image equal reference image. The IQI and AVG of the proposed method are the greatest. Higher AVG value of average gradient shows a higher spatial resolution. Overall cross entropy (OCE), is used to measure the difference between the two source images and the fused image. Small value corresponds to good fusion result obtained. The OCE and RMSE of the new method are least in the three sets. It is shown that, the proposed method gives the best fusion results in the four fused images. It can be easily shown (Figure 4) that image fusion based on wavelet the edges of bones and tissues in human brain are very blurred. The contourlet based method [8], performs better than previous method. However, the best image fusion result is obtained by applying the proposed fusion algorithm. The image contents like tissues are clearly improved. Other useful information like brain boundaries and shape are almost completely preserved in proposed algorithm.

\section{CONCLUSION}

In this paper, a medical image fusion scheme based on shearlet transform and feature visibility is proposed. The experimental results confirmed that the proposed method outperforms the standard fusion methods in the fusion of different medical images. The proposed image fusion algorithm is an effective, efficient, and feasible algorithm. The visual and statistical comparisons demonstrate that the proposed method can preserve the important structure information such as salient feature compared to other methods. This quality makes the proposed method promising applications in medical diagnosis. There are further improvements and extensions that can be achieved such as time complexity of shearlets decomposition and precalculating the filter coefficients. We will effort on other image processing methods using shearlets in our future work.

\section{REFERENCES}

[1] N. Al-Azzawi, H. A. M. Sakim, W. A. K. W. Abdullah et al., "Medical image fusion scheme using complex contourlet transform based on PCA." pp. 5813-5816.
[2] S. F. Nemec, M. A. Donat, S. Mehrain et al., "CT-MR image data fusion for computer assisted navigated neurosurgery of temporal bone tumors," European Journal of Radiology, vol. 62, no. 2, pp. 192-198, 2007.

[3] P. Patias, "Medical imaging challenges photogrammetry," ISPRS Journal of Photogrammetry and Remote Sensing, vol. 56, no. 5-6, pp. 295-310, 2002.

[4] G. Pajares, and J. Manuel de la Cruz, "A wavelet-based image fusion tutorial," Pattern Recognition, vol. 37, no. 9, pp. 1855-1872, 2004.

[5] S. Li, and B. Yang, "Hybrid multiresolution method for multisensor multimodal image fusion," IEEE sensor Journal, vol. 10, no. 9, pp. 1519-1526, 2010.

[6] L. Yang, B. L. Guo, and W. Ni, "Multimodality medical image fusion based on multiscale geometric analysis of contourlet transform," Neurocomputing, vol. 72, no. 1-3, pp. 203-211, 2008.

[7] M. Aguilar, and J. R. New, "Fusion of multi-modality volumetric medical imagery." pp. 1206-1212.

[8] L. Yang, B. L. Guo, and W. Ni, "Multimodality medical image fusion based on multiscale geometric analysis of contourlet transform," Neurocomputing, vol. 72, no. 1-3, pp. 203-211, 2008.

[9] N. Al-Azzawi, H. A. M. Sakim, and W. A. K. W. Abdullah, "An efficient medical image fusion method using contourlet transform based on PCA," ISIEA 2009 IEEE Symposium on Industrial Electronics and Applications, Proceedings. pp. 11-19, 2009.

[10] S. Li, H. Yin, and L. Fang, "Group-sparse representation with dictionary learning for medical image denoising and fusion," Biomedical Engineering, IEEE Transactions on, vol. 59, no. 12, pp. 3450-3459, 2012.

[11] N. Al-Azzawi, and W. A. K. W. Abdullah, "Medical Image Fusion Schemes Using Contourlet Transform and PCA Bases," Image Fusion and Its Applications, Y. Zheng, ed., pp. 93-110, InTech: Janeza Trdine 9, 51000 Rijeka, Croatia, 2011.

[12] A. Toet, L. V. Ruyven, and J. Velaton, "Merging thermal and visual images by a contrast pyramid," Optical Engineering, vol. 28, no. 7, pp. 789-792, 1989.

[13] K. Guo, G. Kutyniok, and D. Labate, "Sparse multidimensional representations using anisotropic dilation and shear operators," Wavelets und Splines (Athens, GA, 2005), G. Chen und MJ Lai, eds., Nashboro Press, Nashville, TN, pp. 189-201, 2006.

[14] D. L. Donoho, "Sparse components of images and optimal atomic decompositions," Constructive Approximation, vol. 17, no. 3, pp. 353-382, 2001.

[15] K. Guo, and D. Labate, "Optimally sparse multidimensional representation using shearlets," SIAM journal on mathematical analysis, vol. 39, no. 1, pp. 298318, 2007.

[16] K. Guo, D. Labate, and W.-Q. Lim, "Edge analysis and identification using the continuous shearlet transform," Applied and Computational Harmonic Analysis, vol. 27, no. 1, pp. 24-46, 2009.

[17] S. Liu, M. Shi, Z. Zhu et al., "Image fusion based on complex-shearlet domain with guided filtering," Multidimensional Systems and Signal Processing, pp. 118, 2015.

[18] F. Sillion, and G. Drettakis, "Feature-based control of visibility error: A multi-resolution clustering algorithm for global illumination." pp. 145-152.

[19] J. W. Huang, Y. Q. Shi, and X. H. Dai, "A segmentationbased image coding algorithm using the features of human vision system," Journal Image Graphics, vol. 4, no. 5, pp. 400-404, 1999. 
[20] N. Al-Azzawi, H. A. M. Sakim, and W. A. K. W. Abdullah, "Fast free-form registration based on kullbackleibler distance for multimodal medical image." pp. 484489.

[21] W. Chan, and J. Sivaswamy, "Segmentation of Text Embedded in Clutter using Local Energy," in Proceeding of the IASTED International Conference on Signal and Image Processing, 1998, pp. 364-368.

[22] S. Li, J. T. Kwok, and Y. Wang, "Multifocus image fusion using artificial neural networks," Pattern Recognition Letters, vol. 23, no. 8, pp. 985-997, 2002.

[23] N. M. Oliver, B. Rosario, and A. P. Pentland, "A Bayesian computer vision system for modeling human interactions," Pattern Analysis and Machine Intelligence, IEEE Transactions on, vol. 22, no. 8, pp. 831-843, 2000.

[24] M. C. Morrone, and D. Burr, "Feature detection in human vision: A phase-dependent energy model," Proceedings of the Royal Society of London B: Biological Sciences, vol. 235, no. 1280, pp. 221-245, 1988.
[25] E. F. Erickson, and R. M. Brown, "Calculation of Fringe Visibility in a Laser-Illuminated Interferometer," J. Opt. Soc. Amer., vol. 57, no. 3, 1967.

[26] P. Burt, "The pyramid as a structure for efficient computation," Multiresolution Image Processing and Analysis, A. Rosenfeld, Ed. Springer-Verlag, Berlin, pp. 6-35, 1984.

[27] Z. Li, Z. Jing, X. Yang et al., "Color transfer based remote sensing image fusion using non-separable wavelet frame transform," Pattern Recognition Letters, vol. 26, no. 13, pp. 2006-2014, 2005.

[28] Z. Wang, and A. C. Bovik, "A universal image quality index," IEEE Signal Processing Letters, vol. 9, no. 3, pp. 81-84, 2002.

[29] G. Piella, and H. Heijmans, "A new quality metric for image fusion." pp. 173-176. 